\title{
Electronic-transport properties of parallel double-ring systems
}

\author{
Youyan Liu and Honglin Wang \\ Department of Physics, South China University of Technology, Guangzhou 510641, China \\ Zhao-Qing Zhang \\ Department of Physics, Hong Kong University of Science and Technology, Clear Water Bay, Hong Kong \\ Xiujun Fu \\ China Center of Advanced Science and Technology (World Laboratory), P. O. Box 8730, Beijing 100080, China \\ and Department of Physics, South China University of Technology, Guangzhou 510641, China \\ (Received 14 August 1995; revised manuscript received 6 October 1995)

\begin{abstract}
We have studied a mesoscopic double-ring system connected in parallel and coupled to two electron reservoirs. The system is composed of one-dimensional ordered chains, and the two rings are threaded by magnetic fluxes $\Phi_{1}$ and $\Phi_{2}$, respectively. In the framework of the tight-binding model, the analytical and numerical calculations show that the transmission coefficient $T$ is periodic in fluxes $\Phi_{1}$ and $\Phi_{2}$ with a period $2 \Phi_{0}, \Phi_{0}=h c / e$. For the system there are resonant states $(T=1)$ and antiresonant states $(T=0)$, the distribution of which displays a very remarkable symmetry.
\end{abstract}

Quantum transport in the mesoscopic systems has been extensively studied both experimentally and theoretically in the last decade. ${ }^{1-12}$ In these mesoscopic systems electron transport is governed by quantum mechanics rather than classical mechanics. At very low temperatures, the scattering of phonons (dephasing scattering) is significantly suppressed, and the phase-coherence length of the electrons becomes large compared to the system dimensions. The mesoscopic system can thus be modeled as a phase-coherent elastic scattering. Furthermore, if one considers the electron as a free particle, the idealized sample becomes an electron waveguide.

For the mesoscopic systems, the theoretical study to date has largely concentrated on the isolated ring and open rings connected via leads to electron reservoirs, both of which are threaded by a magnetic flux $\Phi$. For the isolated ring, the persistent currents are the main subjects. ${ }^{3,4,12}$ As for open ring systems the main interest is to study the relationship between the transmission coefficient $T$ and flux $\Phi$. In open ring systems, the electron reservoirs act as a source of energy dissipation or irreversibility, and all scattering processes in the leads and rings are assumed to be elastic. In this line, up to date the theoretical work has mostly been devoted to the study of open single ring or multiring systems in series, and in the framework of waveguide theory. ${ }^{13-16}$ To the best of our knowledge, there is no work devoted to studying the multiring system in parallel, especially in the framework of the tight-binding model. In this Brief Report, we concentrate on examining the electronic-transport properties of open double-ring systems connected in parallel, which are threaded by magnetic fluxes $\Phi_{1}$ and $\Phi_{2}$ as shown in Fig. 1. In the framework of the tight-binding model, but not in the waveguide approximation, we have calculated the transmission coefficient $T$ and found that $T$ is periodic in flux $\Phi$ threaded by the loop with a period $2 \Phi_{0}, \Phi_{0}=h c / e$ being the elementary flux quantum, but not $\Phi_{0}$ as in the case of double-ring systems connected in series. At the same time, we have found interesting symmetries of resonant $(T=1)$ and antiresonant (full reflection, $T=0$ ) states in the $T-\Phi_{1}-\Phi_{2}$ phase diagram. The whole diagram has a very remarkable symmetric pattern.

In the following, we first give an explicit formula to calculate the transmission coefficient $T$ of the open double-ring system connected in parallel and threaded by magnetic fluxes $\Phi_{1}$ and $\Phi_{2}$. As shown in Fig. 1, the studied mesoscopic system can be reduced to a single "ring" with scatterers in upper and lower arms separately, without magnetic flux threading the ring. For an open single ring without magnetic flux as shown in Fig. 2, if we assume that it and the leads are composed of one-dimensional ordered chains with site en$\operatorname{ergy} \varepsilon$ and transfer integral $J$, and $q$ being the electron wave number in the wire, we have an unitary scattering matrix in the tight-binding model for a junction (three-terminal splitter) located at site $N:^{7,10}$
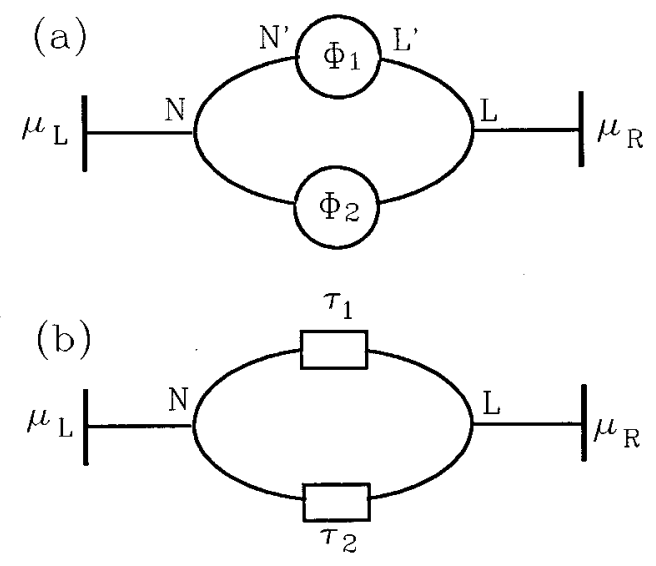

FIG. 1. (a) A parallel double-ring system threaded by magnetic fluxes $\Phi_{1}$ and $\Phi_{2}$, respectively, and connected to two electron reservoirs via ideal leads. (b) Schematic representation for reducing the double-ring system shown in (a) to a single ring with two scatterers in the arms. 


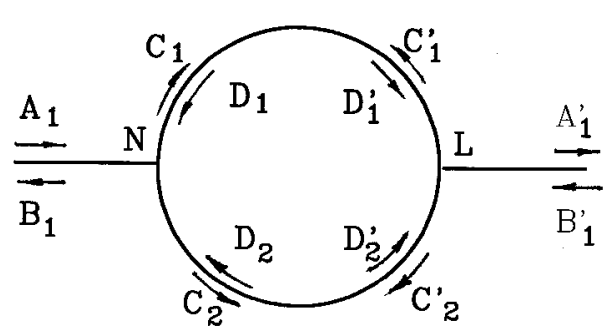

FIG. 2. The relationship of the amplitudes of traveling waves for an open single ring is shown schematically.

$$
S_{N}=\left(\begin{array}{ccc}
c_{N} & \sqrt{K_{N}} & \sqrt{K_{N}} \\
\sqrt{K_{N}} & a_{N} & b_{N} \\
\sqrt{K_{N}} & b_{N} & a_{N}
\end{array}\right),
$$

where

$$
\begin{gathered}
a_{N}=e^{-2 i q N}[2 i J \sin q / D-1], \\
b_{N}=e^{-2 i q N} 2 i J \sin q / D, \\
c_{N}=e^{2 i q N}[2 i J \sin q / D-1], \\
\sqrt{K_{N}}=2 i J \sin q / D, \quad D=E-\varepsilon+3 J e^{i q}, \\
Q=\arccos [-(E-\varepsilon) / 2 J] .
\end{gathered}
$$

Referring to Fig. 2, by the definition of scattering matrix, we have the following equation which relates $S_{N}$ to the amplitudes of traveling waves:

$$
\left(\begin{array}{l}
B_{1} \\
C_{1} \\
C_{2}
\end{array}\right)=S_{N}\left(\begin{array}{c}
A_{1} \\
D_{1} \\
D_{2}
\end{array}\right)
$$

From the above equation, we can obtain

$$
\begin{gathered}
\left(\begin{array}{c}
D_{2} \\
C_{2}
\end{array}\right)=S_{N}^{0}\left(\begin{array}{c}
C_{1} \\
D_{1}
\end{array}\right)+A_{1} V_{N}, \\
B_{1}=\left[c_{N}-\frac{k_{N}}{b_{N}}\right] A_{1}+U_{N}\left(\begin{array}{c}
C_{1} \\
D_{1}
\end{array}\right),
\end{gathered}
$$

where

$$
\begin{gathered}
S_{N}^{0}=\frac{1}{b_{N}}\left(\begin{array}{cc}
1 & -a_{N} \\
a_{N} & b_{N}^{2}-a_{N}^{2}
\end{array}\right), \\
V_{N}=\frac{\sqrt{K_{N}}}{b_{N}}\left(\begin{array}{c}
1 \\
b_{N}-a_{N}
\end{array}\right), \\
U_{N}=\frac{\sqrt{K_{N}}}{b_{N}}\left(1, b_{N}-a_{N}\right) .
\end{gathered}
$$

For the mirror image fork on the right-hand side of the ring, situated at site $L$, the scattering matrix can be obtained by making the following exchanges: $-L \rightarrow N, \quad B_{1}^{\prime} \rightarrow A_{1}$,
$A_{1}^{\prime} \rightarrow B_{1}, C_{2}^{\prime} \rightarrow C_{1}, C_{1}^{\prime} \rightarrow C_{2}, D_{1}^{\prime} \rightarrow D_{2}$, and $D_{2}^{\prime} \rightarrow D_{1}$. In this way, the left-hand side of the ring is described by the analogous equation

$$
\begin{gathered}
\left(\begin{array}{l}
D_{2}^{\prime} \\
C_{2}^{\prime}
\end{array}\right)=S_{-L}^{0}\left(\begin{array}{l}
C_{1}^{\prime} \\
D_{1}^{\prime}
\end{array}\right)+B_{1}^{\prime} V_{-L}, \\
A_{1}^{\prime}=\left(C_{-L}-\frac{k_{-L}}{b_{-L}}\right) B_{1}^{\prime}+U_{-L}\left(\begin{array}{l}
C_{2}^{\prime} \\
D_{2}^{\prime}
\end{array}\right) .
\end{gathered}
$$

$A_{1}^{\prime}$ can be expressed by $A_{1}$ and $B_{1}^{\prime}$ as

$$
\begin{aligned}
A_{1}^{\prime}= & B_{1}^{\prime}\left[C_{-L}-\frac{K_{-L}}{b_{-L}}+U_{-L}\left(\begin{array}{ll}
0 & 1 \\
1 & 0
\end{array}\right) \tau_{1} P\left(\begin{array}{ll}
0 & 1 \\
1 & 0
\end{array}\right) \tau_{2}^{-1} V_{-L}\right] \\
& -A_{1} U_{-L}\left(\begin{array}{ll}
0 & 1 \\
1 & 0
\end{array}\right) \tau_{1} P V_{N}
\end{aligned}
$$

where

$$
P=\left[S_{N}^{0}-\left(\begin{array}{ll}
0 & 1 \\
1 & 0
\end{array}\right) \tau_{2}^{-1} S_{-L}^{0}\left(\begin{array}{ll}
0 & 1 \\
1 & 0
\end{array}\right) \tau_{1}\right]^{-1} .
$$

From Eq. (4), we obtain the transmission amplitude of the double-ring system as

$$
t=-U_{-L}\left(\begin{array}{ll}
0 & 1 \\
1 & 0
\end{array}\right) \tau_{1} P V_{N} .
$$

In the above equations, $\tau_{1}$, and $\tau_{2}$ are the transfer matrices of upper and lower scatterers, respectively. As we have mentioned in the present studied system the scatterers represent the upper and lower rings threaded by magnetic fluxes $\Phi_{1}$ and $\Phi_{2}$, respectively. By definition, $\tau_{1}$ and $\tau_{2}$ satisfy the following relations: ${ }^{10}$

$$
\begin{aligned}
& \left(\begin{array}{l}
D_{1}^{\prime} \\
C_{1}^{\prime}
\end{array}\right)=\tau_{1}\left(\begin{array}{c}
C_{1} \\
D_{1}
\end{array}\right), \quad\left(\begin{array}{l}
D_{2}^{\prime} \\
C_{2}^{\prime}
\end{array}\right)=\tau_{2}\left(\begin{array}{l}
C_{2} \\
D_{2}
\end{array}\right), \\
& \tau_{i}=\frac{1}{t_{i}}\left(\begin{array}{cc}
t_{i}^{2}-r_{i}^{2} & r_{i} \\
-r_{i} & 1
\end{array}\right), \quad i=1 \text { and } 2 .
\end{aligned}
$$

For a single ring threaded by flux $\Phi$, and connected via leads to electron reservoirs, the reflection and transmission amplitudes can be given as follows: ${ }^{10}$

$$
\begin{gathered}
r_{i}=e^{2 i q N_{i}}\left\{C-\frac{2 K}{d_{i}}\left[b \cos \phi_{i}+a-e^{i \psi}\left(b^{2}-a^{2}\right)(b-a)\right]\right\}, \\
t_{i}=2 K\left[\left(\cos \phi_{i} / 2\right) / d_{i}\right]\left[(b-a)^{2}-e^{-i \psi}\right],
\end{gathered}
$$

where

$$
\begin{gathered}
\phi_{i}=2 \pi \Phi_{i} / \Phi_{0}, \\
d_{i}=2 b^{2} \cos \phi_{i}-e^{-i \psi}-\left(b^{2}-a^{2}\right)^{2} e^{i \psi}+2 a^{2}, \\
\psi=2 q(L-N)=q S,
\end{gathered}
$$

and $S$ is the circumference length of the ring. 
What we are mostly interested in is the periodicity of the transmission coefficient $T$. In the following, we denote the parameters of upper and lower rings by indexes 1 and 2, respectively. We now prove that $T$ is periodic in fluxes $\Phi_{1}\left(\Phi_{2}\right)$, if we fix $\Phi_{2}\left(\Phi_{1}\right)$ unchanged, with a period $2 \Phi_{0}$, $\Phi_{0}=h c / e$. Because the calculation is very tedious and straightforward, here we present only some key results. The formula to calculate the transmission amplitude $t$ is formula (5). From Eq. (4), we can obtain that

$$
P=\frac{b t_{1} t_{2}}{F}\left(\begin{array}{cc}
\left(b^{2}-a^{2}\right) t_{1} t_{2}-M_{12} & M_{11}-a t_{1} t_{2} \\
a t_{1} t_{2}+M_{22} & t_{1} t_{2}-M_{21}
\end{array}\right),
$$

where

$$
\begin{gathered}
F=\left(t_{1} t_{2}-M_{21}\right)\left[\left(b^{2}-a^{2}\right) t_{1} t_{2}-M_{12}\right] \\
+\left(a t_{1} t_{2}-M_{11}\right)\left(a t_{1} t_{2}+M_{22}\right), \\
M_{11}=-r_{1} e^{-2 i q L}-a\left(t_{1}^{2}-r_{1}^{2}\right)-a r_{1} r_{2} \\
+e^{2 i q L}\left(b^{2}-a^{2}\right)\left(t_{1}^{2}-r_{1}^{2}\right) r_{2}, \\
M_{12}=e^{-2 i q L}-a r_{1}+a r_{2}+r_{1} r_{2} e^{2 i q L}\left(b^{2}-a^{2}\right), \\
M_{21}=r_{1} r_{2} e^{-2 i q L}+a r_{2}\left(t_{1}^{2}-r_{1}^{2}\right)-a r_{1}\left(t_{2}^{2}-r_{2}^{2}\right) \\
+e^{2 i q L}\left(b^{2}-a^{2}\right)\left(t_{1}^{2}-r_{1}^{2}\right)\left(t_{2}^{2}-r_{2}^{2}\right), \\
M_{22}=-r_{2} e^{-2 i q L}+a r_{1} r_{2}+a\left(t_{2}^{2}-r_{2}^{2}\right) \\
+r_{1} e^{2 i q L}\left(b^{2}-a^{2}\right)\left(t_{2}^{2}-r_{2}^{2}\right) .
\end{gathered}
$$

In this way, we have

$$
t=-\left(b t_{2} / F\right)\left(e^{-2 i q L} m_{2}+m_{1}\right)
$$

where

$$
\begin{aligned}
m_{1}=[ & \left.M_{11}+M_{12}-(3 a+1) t_{1} t_{2}\right]\left(t_{1}^{2}-t_{2}^{2}\right) \\
& +r_{1}\left[(1-a) t_{1} t_{2}-M_{21}-M_{22}\right], \\
m_{2}= & -r_{1}\left[M_{11}+M_{12}-t_{1} t_{2}(3 a+1)\right] \\
& +\left[(1-a) t_{1} t_{2}-M_{21}-M_{22}\right] .
\end{aligned}
$$

From the above results, we can see that the transmission coefficient $T=|t|^{2}$ has a very heavy and complicated expression. Because what we are mostly interested in is the relationship among $T$ and the fluxes $\Phi_{1}$ and $\Phi_{2}$, so we draw out the factors containing fluxes $\Phi_{1}$ and $\Phi_{2}$ from the formula and set the rest of the parameters in other factors by a function form. We have noticed that the total transmission amplitude $t$ is a polynomial expression of the amplitudes $t_{1}, t_{2}$, $r_{1}$, and $r_{2}$ corresponding to the upper and lower rings. By formulas (7) and (8), the explicit relations between $t_{i}, r_{i}$, and $\phi_{i}$ should be as follows:

$$
r_{i}=A_{i}+B_{i} \cos \phi_{i}, \quad t_{i}=C_{i} \cos \left(\phi_{i} / 2\right) /\left(D_{i}+E_{i} \cos \phi_{i}\right),
$$

where $A_{i}, B_{i}, C_{i}, D_{i}$, and $E_{i}$ are the function factors containing other parameters except fluxes $\Phi_{i}$. If we fix the flux $\Phi_{1}$ of the upper ring to show how the $T$ would vary following the change of flux $\Phi_{2}$, we have

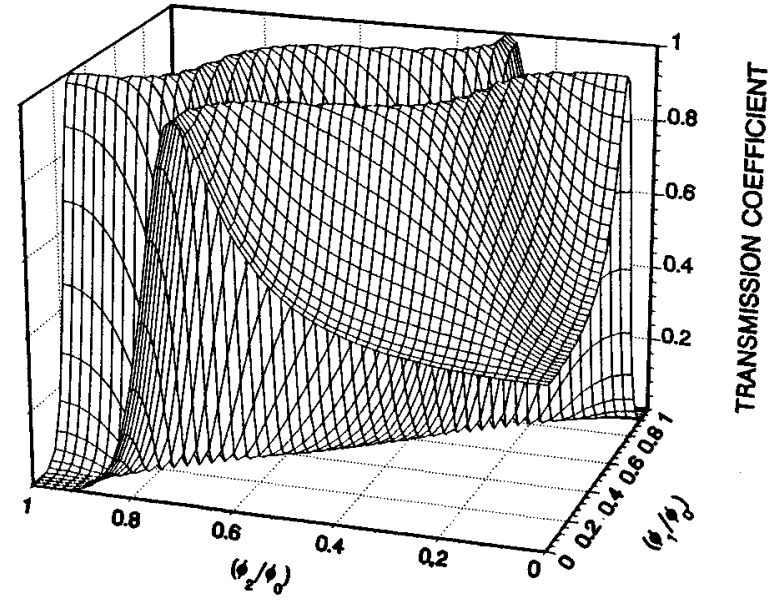

FIG. 3. Transmission coefficient $T$ vs magnetic fluxes $\Phi_{1}$ and $\Phi_{2}$ for the parallel double-ring system shown in Fig. 1. The parameters of the system are as follows: the electron energy $E=1.0$, $\varepsilon=0$, and $J=-1.0$, the lengths $N^{\prime}-N=L-L^{\prime}=2$, and the circumference of the upper and lower rings is 4 . $T$ is periodic in flux $\Phi_{1}\left(\Phi_{2}\right)$ for fixed $\Phi_{2}\left(\Phi_{1}\right)$ with period $2 \Phi_{0}$. Here only a quarter of whole period is shown (see Fig. 4).

$$
T=|t|^{2} \sim\left|A \cos \phi_{2}+B \cos \left(\phi_{2} / 2\right)\right|^{2},
$$

where $\phi_{2}=2 \pi \Phi_{2} / \Phi_{0}$, and $A$ and $B$ are the functions of other parameters including $\cos \phi_{2} \equiv \cos \left(2 \pi \Phi_{2} / \Phi_{0}\right)$, which is periodic in flux $\Phi_{2}$ with a period $\Phi_{0}$, the elementary flux quantum. From expression (10), evidently, $T$ is a period function with period $2 \Phi_{0}$.

Figure 3 shows our numerical results which confirm the above analysis, where we have plotted the transmission coefficient $T$ versus fluxes $\Phi_{1}$ and $\Phi_{2}$ for electrons with energy $E=1.0$, and we have set $\varepsilon=0$ and $J=-1$. From the picture we can see that for a fixed $\Phi_{1}\left(\Phi_{2}\right), T$ is periodic in flux $\Phi_{2}\left(\Phi_{1}\right)$ with a periodic $2 \Phi_{0}$. For the sake of clear visualization we plotted only a quarter of the whole periodic picture, i.e., a $\Phi_{1}$ and $\Phi_{2}$ range from 0 to $\Phi_{0}$, but not $2 \Phi_{0}$. From Fig. 3 we also can see that the $T$ is symmetric for fluxes $\Phi_{1}$ and $\Phi_{2}$. Because if we denote the flux coordinates of point $i$ by $\Phi_{1}^{(i)}$ and $\Phi_{2}^{(i)}$, then for two points symmetric to the $(00,11)$ line we have $\Phi_{1}^{(1)}=\Phi_{2}^{(2)}$ and $\Phi_{2}^{(1)}=\Phi_{1}^{(2)}$ (see Fig. 4), which gives rise to $T^{(1)}=T^{(2)}$, since the upper and lower arms are symmetric for the studied system. The numerical calculation shows that the antiresonant states (full reflection, $T=0$ with accuracy $10^{-14}$ ) appears exactly in the lines $\Phi_{1} / \Phi_{0}+\Phi_{2} / \Phi_{0}=1, \quad \Phi_{1} / \Phi_{0}-\Phi_{2} / \Phi_{0}=1, \quad \Phi_{1} / \Phi_{0}$ $+\Phi_{2} / \Phi_{0}=3$, and $\Phi_{2} / \Phi_{0}-\Phi_{1} / \Phi_{0}=1$, and that the resonant states $(T=1)$ form a sinuous curve; the data show that it is very closed to a sinusoid. These results are schematically shown in Fig. 4, where we can see a symmetric center $O$ for a $2 \Phi_{0}$ period spectrum, which locates at the point $\Phi_{1} / \Phi_{0}=\Phi_{2} / \Phi_{0}=1$. The four lines, which intersect in point $O$ and are denoted by dashed lines, are symmetric axes of $T$. Furthermore, we have noticed that the full reflection lines $(T=0)$ are also symmetric axes. This means that two points locating symmetrically at two sides of the full reflection line have the same $T$. We prove this interesting conclusion as follows. Assume that two points are symmetric to each other by full reflection line $\Phi_{1} / \Phi_{0}+\Phi_{2} / \Phi_{0}=1$, and that the cor- 


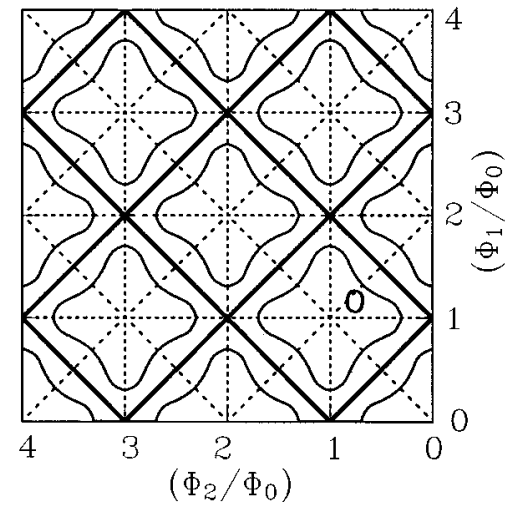

FIG. 4. The symmetrization and periodicity of the transmission coefficient $T$ shown in Fig. 3 are presented schematically. The picture shows four whole periods. The thick lines are antiresonant states with $T=0$, the thin curves are resonant states. All of the dashed lines and thick lines are symmetric axes, which determine variant periodicities of $T$.

responding parameters are $\Phi_{1}^{(1)}, \Phi_{2}^{(1)}$ and $\Phi_{1}^{(2)}, \Phi_{2}^{(2)}$; it is easy to prove that $\Phi_{1}^{(1)} / \Phi_{0}+\Phi_{2}^{(2)} / \Phi_{0}=\Phi_{2}^{(1)} / \Phi_{0}+\Phi_{1}^{(2)} /$ $\Phi_{0}=1$. This is because in the formula to calculate $T$, the flux parameters appear only in the cosine functions, and we have

$$
\begin{aligned}
\cos \left(2 \pi \Phi_{2}^{(2)} / \Phi_{0}\right) & =\cos \left[2 \pi\left(1-\Phi_{1}^{(1)} / \Phi_{0}\right)\right] \\
& =\cos \left(2 \pi \Phi_{1}^{(1)} / \Phi_{0}\right), \\
\cos \left(2 \pi \Phi_{1}^{(2)} / \Phi_{0}\right) & =\cos \left[2 \pi\left(1-\Phi_{2}^{(1)} / \Phi_{0}\right)\right] \\
& =\cos \left(2 \pi \Phi_{2}^{(1)} / \Phi_{0}\right) .
\end{aligned}
$$

The above results show that these two states have the same $T$, because the upper and lower arms are symmetric for the studied system, as we have mentioned for the $(00,11)$ line symmetry. Figure 4 displays a remarkable symmetric pattern where we have drawn a picture with four whole periods. In Fig. 4 the thick lines are antiresonant lines (full reflection) with $T=0$, and the thin curves are resonant lines with $T=1$. All of the dashed and thick lines are symmetric lines. In the horizontal and vertical directions, $T$ has a $2 \Phi_{0}$ periodicity, but from (00) to (11), $T$ also experiences a period

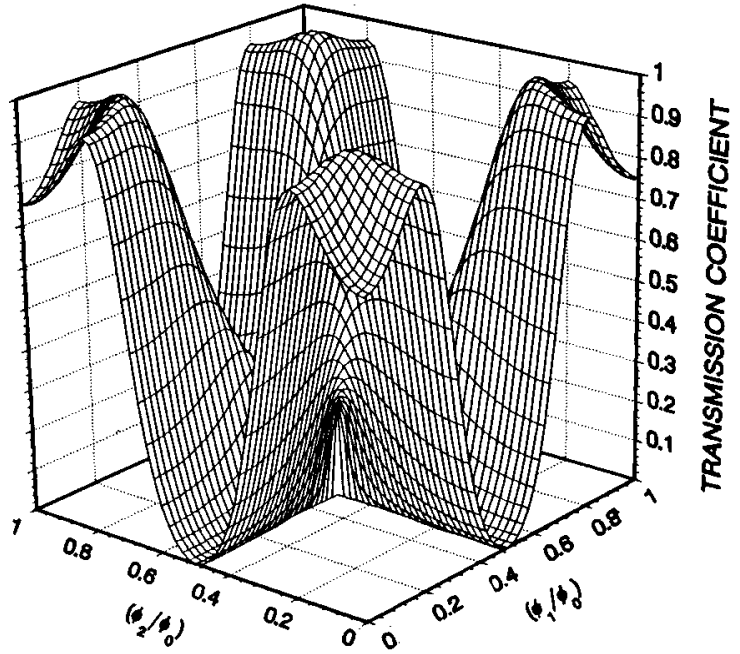

FIG. 5. Transmission coefficient $T$ vs fluxes $\Phi_{1}$ and $\Phi_{2}$ for double-ring systems in series for comparison with Fig. 3. The parameters of the system are same as those of Fig. 3. Evidently, the $T$ is periodic in fluxes $\Phi_{1}$ and $\Phi_{2}$, with period $\Phi_{0}$.

because of the symmetry of antiresonant line $(01,10)$. In the picture there exist other periodicities; for example, in the direction from $(0,1)$ to $(3,2), T$ also experiences a period, in which $\Phi_{1}$ increases by $\Phi_{0}$ and $\Phi_{2}$ by $3 \Phi_{0}$. In some sense, the picture has a periodic-lattice-like periodicity. Here we would like to emphasize that these results for $E=1.0$ are typical; for other energies our calculation shows quite the same structure. Finally, as a comparison, in Fig. 5 we plot the $T$ versus $\Phi$ picture for double-ring systems in series and the same electron energy $E=1.0$ is chosen. We can see that $T$ is periodic in fluxes $\Phi_{1}$ and $\Phi_{2}$ with period $\Phi_{0}$ different from the present systems, where in any case even the flux $\Phi_{2}$ (or $\Phi_{1}$ ) is set zero, the period of $T$ in flux $\Phi_{2}$ (or $\Phi_{1}$ ) is always $2 \Phi_{0}$.

This work was supported by the National Science Foundation of China, Science Foundation of Guangdong Province, and DAG Grant of Hong Kong University of Science and Technology, Grant No. 94/95 SC14.
${ }^{1}$ R. Landauer, Philos. Mag. 21, 863 (1970).

${ }^{2}$ H. L. Engquist and P. W. Anderson, Phys. Rev. B 24, 1151 (1981).

${ }^{3}$ M. Buttiker, Y. Imry, and R. Laudauer, Phys. Lett. 96A, 365 (1983).

${ }^{4}$ Y. Geven, Y. Imry, and M. Ya. Azbel, Phys. Rev. Lett. 52, 129 (1984).

${ }^{5}$ M. Buttiker, R. Landauer, Y. Imry, and S. Pinhas, Phys. Rev. B 31, 6207 (1985).

${ }^{6}$ U. Sivan and Y. Imry, Phys. Rev. B 33, 551 (1986).

${ }^{7}$ O. Entin-Wohlman, C. Hartzstein, and Y. Imry, Phys. Rev. B 34, 921 (1986)

${ }^{8}$ L. P. Levy, G. Dolan, J. Dunsmuir, and H. Bouchiat, Phys. Rev. Lett. 64, 2074 (1990).

${ }^{9}$ P. A. Lee, A. D. Stone, and H. Fukuyama, Phys. Rev. B 35, 1039 (1990)
${ }^{10}$ D. Kowal, U. Sivan, O. Entin-Wohlman, and Y. Imry, Phys. Rev. B 42, 9009 (1990).

${ }^{11}$ Weimin Zhang, Wenji Deng, and Youyan Liu, Chin. Phys. Lett. 11, 357 (1994).

${ }^{12}$ Wenji Deng, Youyan Liu, and Changde Gong, Phys. Rev. B 50, 7655 (1994).

${ }^{13}$ J. Xia, Phys. Rev. B 45, 3593 (1992).

${ }^{14}$ S. Singha Deo and A. M. Jayannavar, Phys. Rev. B 50, 11629 (1994); A. M. Jayannavar and D. Singha Deo, ibid. 51, 10175 (1995).

${ }^{15}$ D. Takai and K. Ohta, Phys. Rev. B 51, 11132 (1995); Phys. Lett. A 199, 401 (1995).

${ }^{16}$ D. Takai and K. Ohta, Phys. Rev. B 50, 2685 (1994); 50, 18250 (1994). 